\title{
EXTREMAL GRAPHS OF DIAMETER 3
}

\author{
L. CACCETTA
}

(Received 28 July 1978)

Communicated by W. D. Wallis

\begin{abstract}
This paper is concerned with graphs of order $n$ and diameter at most 3 having the property that by deleting any $s$ or fewer vertices (edges) the resulting subgraphs (partial graphs) have diameter at most $\lambda$. A graph satisfying the above constraints and having minimum number of edges is said to be extremal. A characterization of extremal graphs is presented for the case $s=1$.
\end{abstract}

Subject classification (Amer. Math. Soc. (MOS) 1970): 05 C 35.

\section{Introduction}

We consider only undirected graphs without loops or multiple edges. The terminology of Bondy and Murty (1976) will be adopted throughout unless stated otherwise.

Denote by $G_{V}(n, k, \lambda, s)$ the class of graphs with $n$ vertices and diameter at most $k$ having the property that by deleting any $s$ or fewer vertices the resulting subgraphs have diameter at most $\lambda \geqslant k$. A graph with minimum number of edges within the class $G_{V}(n, k, \lambda, s)$ is denoted by $\operatorname{Min} G_{V}(n, k, \lambda, s)$ and the minimum possible number of edges is denoted by $M_{V}(n, k, \lambda, s)$. Similarly the class $G_{E}(n, k, \lambda, s)$ consists of those graphs with $n$ vertices and diameter at most $k$ having the property that by deleting any $s$ or fewer edges the resulting partial graphs have diameter at most $\lambda \geqslant k$. A graph with the minimum possible number of edges within the class $G_{E}(n, k, \lambda, s)$ is denoted by $\operatorname{Min} G_{E}(n, k, \lambda, s)$, and the minimum possible number of edges is denoted by $M_{E}(n, k, \lambda, s)$. The graphs with the minimal number of edges within these classes will be called extremal graphs.

Several papers have appeared in the literature on this problem. In Caccetta (1978) we presented a comprehensive bibliography of all pertinent developments. 
An earlier paper by Bollobás and Harary (1976) also provides an exposition of some of the known results. In this paper we are concerned only with the case of diameter 3 , that is $k=3$. For $s>1$ some asymptotic results are known (see Bollabás (1976) and Caccetta (1978)); in particular

$$
\lim _{n \rightarrow \infty} \frac{M_{\mathcal{V}}(n, 3, n-1, s)}{n}=\frac{1}{2}\left\{(s+1)+\frac{1}{s+1}\right\},
$$

and for $\lambda \geqslant 4$

$$
\lim _{n \rightarrow \infty} \frac{M_{E}(n, 3, \lambda, s)}{n}=\frac{1}{2}(s+2) .
$$

For $s=1$ the functions $M_{V}(n, 3, \lambda, 1), \lambda \geqslant 4$, and $M_{E}(n, 3, \lambda, 1), \lambda \geqslant 6$ were determined by Bollobás (1968a, b). Apart from determining $M_{E}(n, 3, \lambda, 1)$ for $\lambda=4$ and 5 , which was previously unknown, the main contribution of this paper is the characterization of the extremal graphs. The case $\lambda=3$ was studied in Caccetta (1976a) and so in the following we consider only the case $\lambda \geqslant 4$.

\section{Preliminaries}

Throughout this paper $G$ always denotes an extremal graph with $M$ edges; the class of graphs for which $G$ is a member will be clear by the context in which $G$ is used. The diameter of $G$ is denoted by $D(G)$. We denote by $V(G)(E(G))$ the vertex (edge) set of $G$. For any set $S$ of vertices or edges we denote by $|S|$ the number of elements in $S$. The minimum degree of $G$ is denoted by $\mu(G)$. We denote by $\alpha$ any vertex of $G$ having minimum degree. $P, Q$ and $R$ denote the subgraphs of $G-\alpha$ whose vertices are at distance 1,2 and 3, respectively, from $\alpha$ in $G$. If $R \neq \varnothing$ we let $R_{1}, R_{2}, \ldots, R_{p}$ denote the components of $R$. Denote by:

$A$ : those vertices of $Q$ that are adjacent to at least two vertices of $P$ or $Q$.

$W$ : those vertices of $Q-A$ that are adjacent to exactly one other vertex of $Q$.

$U:$ the vertices of $Q-A-W$.

$Q_{i}$ : those vertices of $Q$ that are adjacent to vertices of $R_{i}$.

$A_{i}, W_{i}$ and $U_{i}$ : those vertices of $Q_{i}$ that are in $A, W$ and $U$, respectively.

$Q^{*}$ : the subgraph spanned by the vertices of $Q$ that are not adjacent to any vertex of $R$.

$e_{i}$ : the number of edges in $R_{i}$.

$f_{i}$ : the number of edges connecting $R_{i}$ to $Q$.

$[x]$ : integer part of $x$.

For each set of vertices defined above the corresponding lower case letter will always denote the number of vertices in that set, for example $a$ denotes the number of vertices in $A$. 
We conclude this section with the following rather obvious observations:

(1) A $G_{V}(n, k, \lambda, s)\left(G_{E}(n, k, \lambda, s)\right)$ graph is also a $G_{V}\left(n, k^{\prime}, \lambda^{\prime}, s^{\prime}\right)\left(G_{E}\left(n, k^{\prime}, \lambda^{\prime}, s^{\prime}\right)\right)$ graph, whenever $k^{\prime} \geqslant k, \lambda^{\prime} \geqslant \lambda$ and $s^{\prime} \leqslant s$. Consequently the functions $M_{V}(n, k, \lambda, s)$ and $M_{E}(n, k, \lambda, s)$ are monotonic non-decreasing in $s$, and monotonic non-increasing in $k$ and $\lambda$.

(2) In a $G_{V}(n, k, \lambda, s)\left(G_{E}(n, k, \lambda, s)\right)$ there will be at least $s+1$ vertex (edge) disjoint paths of length $\leqslant \lambda$ between any two non-adjacent (any two) vertices, at least one of which has length $\leqslant k$.

(3) The degree of every vertex of $G$ is at least $s+1$, that is, $\mu(G) \geqslant s+1$.

(4) If $\mu(G)=s+1$, then every vertex of $G$ which is not adjacent to $\alpha$ must be connected to each of the $s+1$ vertices adjacent to $\alpha$ by a path of length $\leqslant \lambda-1$ (from observation (2)).

\section{The structures of $\operatorname{Min} G_{\nu}(n, 3,4,1)$}

Denote by $K^{2 m}$ the class of graphs on $2 m(\geqslant 6)$ vertices that are obtained by connecting two vertices $x_{1}$ and $x_{4}$ by $m$ disjoint paths of length 3 . The class $K^{2 m+1}$ is obtained from the class $K^{2 m}$ by adding one vertex and connecting it to any two non-adjacent vertices of $K^{2 m}$. Clearly the graphs of $K^{2 m}$ and $K^{2 m+1}$ belong to the class $G_{V}(n, 3,4,1)$ and they have $\left[\frac{1}{2}(3 n-5)\right]$ edges (see Figure 1; we have labelled the vertex $x_{i}$ as $i$ ). Note that there are 3 members $K_{j}^{2 m+1}, j=1,2,3$, of the class $K^{2 m+1}$. Bollobás (1968a) conjectured that these are the only extremal graphs of $G_{V}(n, 3,4,1)$. We shall prove this is indeed the case.
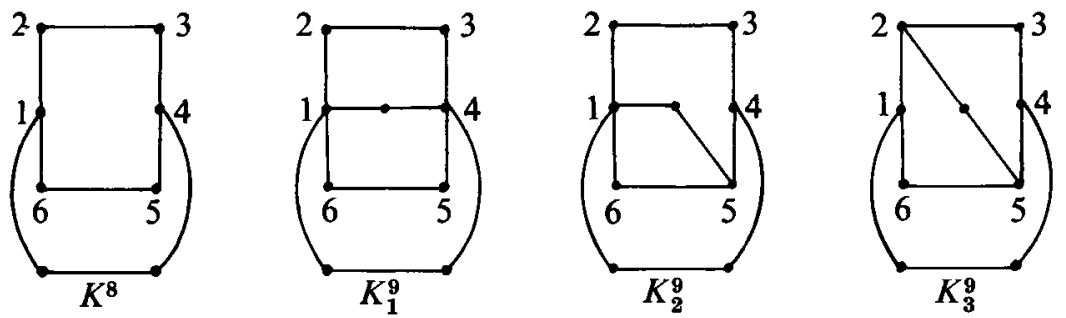

FIGURE 1.

The graphs of $K^{2 m}$ and $K^{2 m+1}$ have one common feature, namely two adjacent vertices of degree 2 . We shall prove that every $G$ has two adjacent vertices of degree 2 for all $n \geqslant 6$ except for $n=9$, and furthermore for $n=9$ the only other possible graph is the graph $H^{9}$ of Fig. 2 . Once this is established we can easily obtain the extremal structures because of the following lemma. 


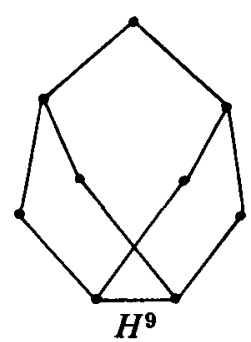

FIGURE 2.

LEMMA 1. If $G$ possesses two adjacent vertices of of degree 2 , then

$$
M_{V}(n, 3,4,1)=\left[\frac{1}{2}(3 n-5)\right],
$$

and the graphs of $K^{2 m}$ and $K^{2 m+1}$ are the only elements of $\operatorname{Min} G_{V}(n, 3,4,1)$ for $n=2 m$ and $n=2 m+1$, respectively.

Proof. Let $\alpha$ and $\beta$ denote any two adjacent vertices of $G$ having degree 2 and suppose $\alpha(\beta)$ is adjacent to $x(y)$. We adopt the notation of the previous section with one minor modification. Here we let $Q$ denote the subgraph of $G-\alpha-\beta$ whose vertices are adjacent to at least one of $x$ and $y$, and denote by $R$ the subgraph formed by the remaining vertices. Every vertex of $R \cup Q$ must be connected by a path of length $\leqslant 2$ to each of $x$ and $y$ (by observation (4) of Section 2). Hence $U=\emptyset$ and every vertex of $R$ is adjacent to at least two vertices of $Q$. Therefore simply by counting the number of edges:

$$
M \geqslant 3+2 a+\frac{3}{2} w+2 r+\varepsilon=\frac{1}{2}(3 n-6)+\frac{1}{2}(a+r+2 \varepsilon),
$$

where

$$
\varepsilon= \begin{cases}1, & \text { if } x \text { and } y \text { are adjacent, } \\ 0, & \text { otherwise. }\end{cases}
$$

Clearly $\varepsilon=0$ and $a+r \leqslant 1$ for otherwise $M>(3 n-5) / 2$. Now $M=\frac{1}{2}(3 n-6)$ only if $A \cup R=\emptyset$ and $n$ is even, giving the structures $K^{2 m}, n=2 m$. If $n$ is odd, $M=\frac{1}{2}(3 n-5)$ only if $a+r \leqslant 1$, hence the structures $K^{2 m+1}, n=2 m+1$. This completes the proof of Lemma 1.

Before proceeding to prove the existence of two adjacent vertices by degree 2 in $G$ we introduce some further notation. Denote by:

$R^{(1)}$ : those vertices of $R$ that are adjacent to at least two vertices of $Q$.

$R^{(2)}$ : the vertices of $R-R^{(1)}$.

$R_{i}^{(1)}$ and $R_{i}^{(2)}$ : the vertices of $R_{i} \cap R^{(1)}$ and $R_{i} \cap R^{(2)}$, respectively.

$V_{i}$ : those vertices of $U_{i}$ that are adjacent to at least one vertex of $R_{i}^{(1)}$.

For each of the above sets the corresponding lower case letter will denote the number of elements in that set. 
The following observations are easily made.

(1) $\mu(G)=2$ since $M<3 n / 2$ (by the above constructions). We let $P=\{x, y\}$.

(2) Every vertex of $Q \cup R$ should be connected by a path of length $\leqslant 3$ to each of $x$ and $y$ (by observation (4) of Section 2). Hence every vertex of $U$ must be adjacent to at least one vertex of $R^{(1)}$.

(3) If $R=\varnothing$ then $G$ possesses two adjacent vertices of degree 2 for otherwise $Q=A$ and hence $M \geqslant 2 n-4>\frac{1}{2}(3 n-5)$.

As the aim is to prove the existence of two adjacent vertices of degree 2 , let us suppose that no two adjacent vertices in $G$ have degree 2 . Then from observation (3) above $R \neq \varnothing$.

For a connected component $R_{i}$, we have $e_{i} \geqslant r_{i}-1, f_{i} \geqslant \max \left\{q_{i}, r_{i}+r_{i}^{(1)}\right\}$ and hence

$$
e_{i}+f_{i} \geqslant \frac{3}{2} r_{i}+\frac{1}{2} v_{i}+\frac{1}{2}\left\{\left(q_{i}-v_{i}\right)+r_{i}^{(1)}+\left|q_{i}-r_{i}-r_{i}^{(1)}\right|-2\right\} .
$$

Thus we have

LemMa 2. For every $i=1,2, \ldots, p$ we have

$$
d_{i}=e_{i}+f_{i}-\frac{3}{2} r_{i}-\frac{1}{2} v_{i} \geqslant \frac{1}{2}\left\{\left(q_{i}-v_{i}\right)+r_{i}^{(1)}+\left|q_{i}-r_{i}-r_{i}^{(1)}\right|-2\right\}=\delta_{i} .
$$

Observe that $d_{i} \geqslant-\frac{1}{2}$, since if $r_{i}^{(1)}=0, v_{i}=0$ and $q_{i} \geqslant 2$. Also, if $d_{i}>-\frac{1}{2}$ then $d_{i} \geqslant 0$.

The following are three useful corollaries of Lemma 2.

COROLLARY 1. If $d_{i}=-\frac{1}{2}$, then $r_{i}^{(1)}=r_{i}=1$ and $q_{i}=v_{i}=2$.

COROLlaRY 2. If $d_{i}=0$, then either

(a) $r_{i}^{(1)}=1$ and one of the following conditions is satisfied:

(i) $v_{i}=2, r_{i}=2$ and $q_{i}=2$ or 3 ,

(ii) $v_{i}=q_{i}=3$ and $r_{i}=1$,

(iii) $v_{i}=r_{i}=w_{i}=1$ and $q_{i}=2$,

or

(b) $r_{i}^{(1)}=2, q_{i}=v_{i}=4$ and $r_{i}=2$.

COROLLARY 3. If $d_{i}=\frac{1}{2}$, then one of the following must hold:

(a) $r_{i}^{(1)}=0$ and $q_{i}=r_{i}=3$ or $q_{i}=r_{i}-1=2$.

(b) $r_{i}^{(1)}=1$ and one of the following conditions holds:

(i) $v_{i}=2, r_{i}=3$ and $q_{i}=2,3$ or 4

(ii) $v_{i}=2, r_{i}=1$ and $q_{i}=3$

(iii) $v_{i}=w_{i}=1, r_{i}=2$ and $q_{i}=3$

(iv) $v_{i}=0, r_{i}=1$ and $q_{i}=2$. 
(c) $r_{i}^{(1)}=2$ and one of the following conditions holds:

(i) $q_{i}=v_{i}=3$ and $r_{i}=2$

(ii) $q_{i}=v_{i}=4$ and $r_{i}=3$

(iii) $q_{i}=5, v_{i}=4$ and $r_{i}=3$

(d) $r_{i}^{(1)}=3$ and $q_{i}=v_{i}=6$.

The above corollaries are easily established from Lemma 2.

LEMMA 3. $G$ possesses two adjacent vertices of degree 2 for every $n \geqslant 5$ except possibly $n=9$ for which case the only other possible graph is the graph $H^{9}$ of Figure 2 .

Proof. Simply by counting the number of edges:

$$
M \geqslant 2+2 a+\frac{3}{2} w+u+\varepsilon+\sum_{i=1}^{p}\left(e_{i}+f_{i}\right),
$$

where $\varepsilon$ is defined as in the proof of Lemma 1 .

Since every vertex of $U$ is adjacent to at least one vertex of $R^{(1)}$ we must have

$$
\sum_{i=1}^{p} v_{i} \geqslant u
$$

Note that if $V_{i} \cap V_{j} \neq \varnothing$ for any $i \neq j$, then strict inequality holds in (5). Equations (2), (4) and (5) give:

$$
M \geqslant 2+\frac{3}{2}(n-3)+\frac{1}{2} a+\varepsilon+\sum_{i=1}^{p} d_{i}=\frac{1}{2}(3 n-5)+\frac{1}{2} a+\varepsilon+\sum_{i=1}^{p} d_{i}
$$

Now if $\frac{1}{2} a+\varepsilon+\sum_{i=1}^{p} d_{i} \geqslant \frac{1}{2}$, then obviously $M>\frac{1}{2}(3 n-5)$. Therefore, since $M \leqslant \frac{1}{2}(3 n-5)$ (by above constructions), we may suppose that

$$
\frac{1}{2} a+\varepsilon+\sum_{i=1}^{p} d_{i}<\frac{1}{2}
$$

Let

$$
d_{1}=\min _{1 \leqslant i \leqslant p}\left\{d_{i}\right\}
$$

Obviously $d_{1}=0$ or $-\frac{1}{2}$. We consider the two cases separately.

Case (a) $d_{1}=0$.

Clearly for (7) to hold we must have $A=\varnothing, \varepsilon=0, d_{i}=0$ for every $i=1,2, \ldots, p$ and $V_{i} \cap V_{j}=\varnothing$ for every $i \neq j$. So every component $R_{i}$ of $R$ must satisfy one of the conditions of Corollary 2 of Lemma 2. If $p=1$, then condition (a) of that corollary can be discarded, since in all cases one of $x$ or $y$ has degree 2 in which case $G$ would have two adjacent vertices of degree 2 . In the other case we obtain the graph $H^{9}$. If $p>1$, then it is easily checked that the diameter constraints are violated under the assumption that $V_{i} \cap V_{j}=\varnothing$ for every $i \neq j$. 
Case (b) $d_{1}=-\frac{1}{2}$.

If $p=1$, then obviously $G$ has adjacent vertices of degree 2 , hence $p \geqslant 2$. By Corollary 1 of Lemma 2 we must have $v_{1}=q_{1}=2$ and $r_{1}=1$. For every $i \geqslant 2$, $Q_{1} \cap Q_{i} \neq \varnothing$ since $D(G) \leqslant 3$. Hence if $d_{i}=-\frac{1}{2}$, then $V_{1} \cap V_{i} \neq \varnothing$ since $Q_{i}=V_{i}$. Also, if $d_{i}=0, i \geqslant 2$, then $V_{1} \cap V_{i} \neq \varnothing$ to provide for an alternative path of length $\leqslant 4$ between the vertices of $R_{1}$ and $R_{i}$ when we delete the vertex of $V_{1} \cap Q_{i}$ (note that $r_{i} \leqslant 2$ and $r_{i}^{(1)} \geqslant 1$, since $\left.d_{i}=0\right)$. If $d_{i}=\frac{1}{2}, i \geqslant 2$, then either $V_{1} \cap V_{i} \neq \varnothing$ or else $\left|V_{1} \cap Q_{i}\right|=2, r_{i}=3$ and $r_{i}^{(1)}=0$ or 1 for otherwise the diameter constraints are violated.

Let $p_{1}, p_{2}, p_{3}$ and $p_{4}$ denote the number of components of $R$ with $d_{i}=-\frac{1}{2}, 0, \frac{1}{2}$ and $>\frac{1}{2}$ respectively. For $i \geqslant 2$ we let $V_{i}^{\prime}=V_{i}-V_{i} \cap V_{1}$, and for $i \neq j \geqslant 2$ we let $b_{i j}=\left|V_{i}^{\prime} \cap V_{j}^{\prime}\right|$. It follows from the above that

$$
\sum_{i=1}\left(v_{i}+d_{i}\right) \geqslant u+p_{2}+p_{3}+2 p_{4}-1+\sum_{2 \leqslant i \neq j \leqslant p} b_{i j} .
$$

Equations (2), (4) and (8) give:

$$
M \geqslant \frac{1}{2}(3 n-5)+\frac{1}{2}\left\{a+2 \varepsilon+p_{2}+p_{3}+2 p_{4}-1+\sum_{2 \leqslant i \neq j \leqslant p} b_{i j}\right\} .
$$

If $A \neq \varnothing$, then $M=\frac{1}{2}(3 n-5)$ only if $a=1, \varepsilon=0, p_{2}=p_{3}=p_{4}=0$ and $\sum_{2 \leqslant i \neq j \leqslant p} b_{i j}=0$, that is, only if $G$ has adjacent vertices of degree 2 . Therefore we may suppose that $A=\varnothing$. If $n$ is even, then clearly $M=\frac{1}{2}(3 n-6)$ only if $G$ has adjacent vertices of degree 2 . So the only case that needs to be considered is the case $n$ odd. Now clearly $M=\frac{1}{2}(3 n-5)$ only if

$$
\varepsilon=0, \quad p_{4}=0 \text { and } p_{2}+p_{3}+\sum_{2 \leqslant i \neq j \leqslant p} b_{i j} \leqslant 1 \text {. }
$$

Since $x$ and $y$ are not adjacent $(\varepsilon=0)$ every component $R_{i}$ with $d_{i}=-\frac{1}{2}$ must have one vertex of $V_{i}$ adjacent to $x$ and the other to $y$ in order to provide for an alternative path of length $\leqslant 4$ between $\alpha$ and the vertex of $R_{i}$ when we delete one of $x$ or $y$. Two possibilities arise:

(i) $p_{2}+p_{3}=1$. Then $b_{i j}=0$ for every $i, j \geqslant 2, i \neq j$. Suppose $d_{p}>-\frac{1}{2}$. Then $d_{p}=0$ or $\frac{1}{2}$. If $d_{p}=0$, then $r_{p} \leqslant 2$ and $q_{p} \leqslant 3$ (by Corollary 2 of Lemma 2). The lemma is obvious if $p \geqslant 3$, hence we suppose $p=2$. Then $\left|V_{1} \cap Q_{p}\right|=2$ for otherwise we have adjacent vertices of degree 2 . But then $q \leqslant 3$, and hence one of $x$ or $y$ has degree 2 . Suppose now that $d_{p}=\frac{1}{2}$. Clearly $V_{p} \cap V_{i}=\emptyset$ for every $i \neq p$ for otherwise strict inequality holds in (8). Therefore $\left|V_{1} \cap Q_{p}\right|=2$. Since $d_{p}=\frac{1}{2}, R_{p}$ must be a tree and $r_{p}=r_{p}^{(2)}=3$. Consequently at least one vertex, $s$ say, of $V_{1}$ must be adjacent to a vertex, $t$ say, of $R_{p}$ having degree 2 . But then there is no $(t, x)$-path of length $\leqslant 4$ when we delete the vertex $s$, hence the lemma is proved for this case.

(ii) $p_{2}=p_{3}=0$. Then $\sum_{2 \leqslant i \neq j \leqslant p} b_{i j} \leqslant 1$ and $d_{i}=-\frac{1}{2}$ for every $i=1,2, \ldots, p$. $V_{i} \cap V_{j} \neq \varnothing$ for every $i \neq j$ since $D(G) \leqslant 3$. Now $q \geqslant 4$ for otherwise one of $x$ or $y$ has 
degree 2 . Consequently $G$ must have adjacent vertices of degree 2 as otherwise

$$
\sum_{2 \leqslant i \neq j \leqslant p} b_{i j} \geqslant 2 \text {. }
$$

This completes the proof of Lemma 3.

Lemmas 1 and 3 give:

THEOREM 1. For every $n \geqslant 5, M_{V}(n, 3,4,1)=\left[\frac{1}{2}(3 n-5)\right]$ and the graphs of $H^{9}$ $K^{2 m}$ and $K^{2 m+1}$ are elements of $\operatorname{Min} G_{V}(n, 3,4,1)$ for $n=9, n=2 m$ and $n=2 m+1$, respectively.

\section{The structures of $\operatorname{Min} G_{V}(n, 3, \lambda, 1), \lambda \geqslant 5$}

Bollobás (1968a) showed that $M_{V}(n, 3, \lambda, 1)=\left[\frac{1}{2}(3 n-6)\right], \lambda \geqslant 5$. In this section we confirm this result and the structures of $\operatorname{Min} G_{V}(n, 3, \lambda, 1)$.

Denote by $L_{1}^{2 m+1}$ the class of graphs on $2 m+1$ vertices $(m>3)$ obtained from the class $K^{2 m}$ (defined in the previous section) by inserting a vertex $x_{7}$ along the edge $x_{1} x_{6}$. The class $L_{2}^{2 m+1}$ is obtained from the class $L_{1}^{2 m^{\prime}+1}\left(m^{\prime}<m\right)$ by adding $m-m^{\prime}$ disjoint $\left(x_{1}, x_{5}\right)$-paths of length 3 . We form the class $L_{1}^{2 m}$ from the class $L_{1}^{2 m-3}$ by adding 3 vertices, $x_{2 m-2}, x_{2 m-1}$ and $x_{2 m}$ together with the edges $x_{1} x_{2 m-2}$, $x_{2 m-2} x_{2 m-1}, x_{2 m-1} x_{2 m}, x_{2 m} x_{4}$ and $x_{2 m-1} x_{6}$ or $x_{2 m-1} x_{5}$. The class $L_{2}^{2 m}$ is formed from the class $L_{1}^{2 m-1}$ or $L_{2}^{2 m-1}$ by adding one vertex $x_{2 m}$ and connecting it to $x_{1}$ and any one of the vertices $x_{3}, x_{4}, x_{5}$ or $x_{6}$. The class $L_{3}^{2 m}$ is obtained from the class $L_{1}^{2 m-1}$ by adding a vertex $x_{2 m}$ and connecting it to $x_{7}$ and any one of the vertices $x_{3}, x_{4}$ or $x_{5}$. For $m \geqslant 4$ put $L_{4}^{2 m}=K^{2 m}$. We illustrate some of these constructions in Figure 3.
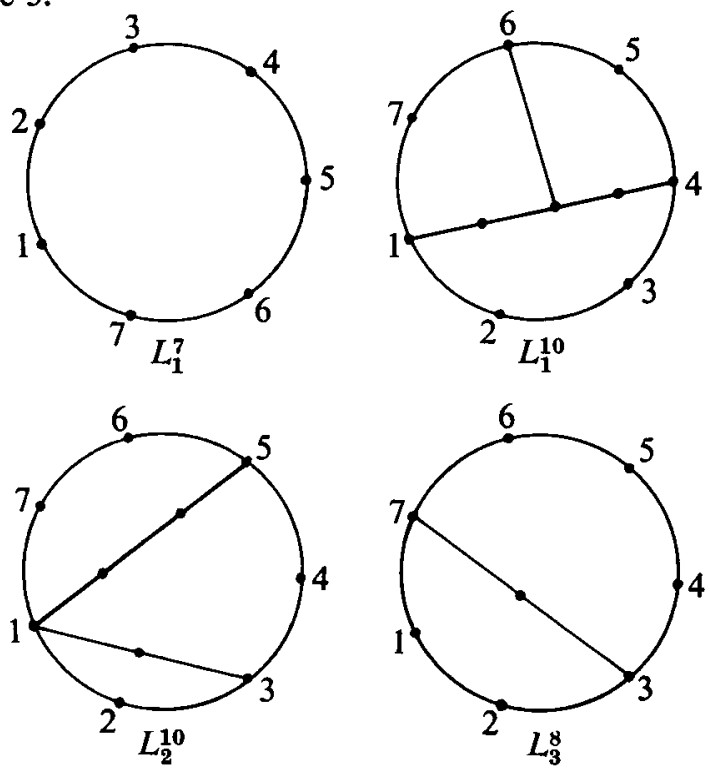

Figure 3. 
It is easily seen that the graphs of $L_{j}^{2 m+1}(j=1,2)$ and $L_{j}^{2 m}(j=1,2,3,4)$ belong to the class $G_{V}(n, 3, \lambda, 1), \lambda \geqslant 5$, for $n=2 m+1$ and $n=2 m$, respectively, and they have $\left[\frac{1}{2}(3 n-6)\right]$ edges (see Figure 3$)$. We shall prove that these graphs together with the graph $H^{12}$ of Figure 4 are the elements of $\operatorname{Min} G_{V}(n, 3, \lambda, 1), \lambda \geqslant 5$, for every $n \geqslant 6$. To do this we need the following lemma.

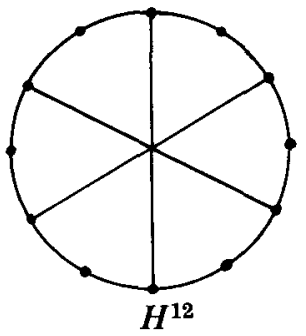

FIGURE 4.

Lemma 4. $G$ possesses two adjacent vertices of degree 2 for every $n \geqslant 6$, except possibly for $n=12$ for which case the only other possible graph is the graph $H^{12}$ of Figure 4.

Replacing $v_{i}$ by $u_{i}$ in (3) and noting that $d_{i}$ may now take on the value -1 (unlike the case $\lambda=4$ it is not necessary that every vertex of $U$ be adjacent to a vertex of $R^{(1)}$ ), we can establish Lemma 4 by using arguments similar to those in the proof of Lemma 3. He omit the details here, but full details can be found in Caccetta (1976b).

THEOREM 2. If $\lambda \geqslant 5$ and $n \geqslant 6$, then $M_{V}(n, 3, \lambda, 1)=\left[\frac{1}{2}(3 n-6)\right]$ and the graphs of $L_{j}^{2 m+1}(j=1,2)$ and $L_{j}^{2 m}(j=1,2,3,4)$ are the elements of $\operatorname{Min} G_{V}(n, 3, \lambda, 1)$ for $n=2 m+1$ and $n=2 m$, respectively.

Proof. By Lemma 4, $G$ has two adjacent vertices, $\alpha$ and $\beta$ say, of degree 2. We adopt the notation in the proof of Lemma 1 . Since $D(G) \leqslant 3$ every vertex of $R_{i}$ must be adjacent to at least two vertices of $Q$, hence

$$
f_{i} \geqslant \max \left\{2 r_{i}, q_{i}\right\}=\frac{1}{2}\left(2 r_{i}+q_{i}\right)+\frac{1}{2}\left|q_{i}-2 r_{i}\right| .
$$

If $U=\varnothing$, then simply by counting the number of edges

$$
M \geqslant 3+2 a+\frac{3}{2} w+2 r=\frac{1}{2}(3 n-6)+\frac{1}{2}(a+r) .
$$

Therefore $M=\left[\frac{1}{2}(3 n-6)\right]$ only if $n$ is even and $A \cup R=\varnothing$, giving the structures $L_{4}^{2 m}, n=2 m$. 
We now consider the case $U \neq \varnothing$ and hence $R \neq \varnothing$. Clearly each component $R_{i}$ of $R$ satisfies the inequality,

$$
e_{i}+f_{i} \geqslant \frac{3}{2} r_{i}+\frac{1}{2} u_{i}+\delta_{i}
$$

where

$$
\delta_{i}=\frac{1}{2}\left\{\left(q_{i}-u_{i}\right)+\left|q_{i}-2 r_{i}\right|+r_{i}-2\right\}
$$

Let

$$
\delta_{1}=\min _{1 \leqslant i \leqslant p}\left\{\delta_{i}\right\}
$$

Simply by counting the number of edges:

$$
M \geqslant 3+2 a+\frac{3}{2} w+u+\sum_{i=1}^{p}\left(e_{i}+f_{i}\right) .
$$

If $\delta_{1}>0$, then (10) and (12) give $M \geqslant \frac{1}{2}(3 n-5)$, hence $\delta_{1} \leqslant 0$. Two cases arise.

Case $(a) . \delta_{1}=0$.

Then

$$
\sum_{i=1}^{p}\left(u_{i}+\delta_{i}\right) \geqslant u .
$$

Note that strict inequality holds in (13) if either $U_{i} \cap U_{j} \neq \varnothing$ for some $i \neq j$ or $\delta_{i}>0$ for some $i \geqslant 2$. Now equations (10), (12) and (13) give $M \geqslant \frac{1}{2}(3 n-6)$, with strict inequality holding if $A \neq \varnothing$ or one of (12) or (13) holds with strict inequality. Therefore, as $D(G) \leqslant 3$, the only possibility is $p=1$ and $n$ even, giving the structures $L_{1}^{2 m}$ (when $r_{1}=2$ and $q_{1}=u_{1}=4$ ) and $L_{2}^{2 m}$ (when $r_{1}=1$ and $q_{1}=u_{1}=3$ ).

Case $(b) . \delta_{1}=-\frac{1}{2}$.

Then $r_{1}=1$ and $q_{1}=u_{1}=2$. $U_{1} \cap U_{i} \neq \varnothing$ for every $i \geqslant 2$ since $D(G) \leqslant 3$. Consequently

$$
\sum_{i=1}^{p}\left(u_{i}+\delta_{i}\right) \geqslant u-2+p_{1}+2 p_{2}+3 p_{3},
$$

where $p_{1}, p_{2}$ and $p_{3}$ denote the number of components with $\delta_{i}=0, \frac{1}{2}$ and $\geqslant 1$, respectively. Clearly $p_{2}=p_{3}=0$ and $p_{1} \leqslant 1$ for otherwise $M \geqslant \frac{1}{2}(3 n-5)$. Therefore $\delta_{i}=-\frac{1}{2}$ for all except possibly one $i, i=1,2, \ldots, p$.

If $p_{1}=0$, then equality in (14) holds only if $\left|U_{1} \cap U_{i}\right|=1$ for every $i \geqslant 2$ and $U_{i}^{\prime} \cap U_{j}^{\prime}=\varnothing$ for every $i, j \geqslant 2, i \neq j$, where $U_{i}^{\prime}=U_{i}-U_{i} \cap U_{1}$. Equations (10), (12) and (14) give:

$$
M \geqslant \frac{1}{2}(3 n-7)+\frac{1}{2} a \text {. }
$$


Now $M=\frac{1}{2}(3 n-7)$ only if $A=\varnothing$ and strict equality holds in (12) and (13). Hence when $n$ is odd the only possible structures are the graphs $L_{1}^{2 m+1}$ (if $p=1$ ) and $L_{2}^{2 m+1}$ (if $p>1$ ), $n=2 m+1$.

Clearly $M=\frac{1}{2}(3 n-6)$ only if $a+p_{1} \leqslant 1$, and if $a+p_{1}=1$ then $U_{i}^{\prime} \cap U_{j}^{\prime}=\varnothing$ for every $i \neq j \geqslant 2$ and $\left|U_{1} \cap U_{i}\right|=1$ for every $i \geqslant 2$. When $a+p_{1}=1$ we get the structures $L_{2}^{2 m}, n=2 m$. When $a=p_{1}=0$ we simply have two vertices of $R$ connected to the same two vertices of $Q$ thus giving the structures $L_{2}^{2 m}$ (if $W=\varnothing$ ) and $L_{3}^{2 m}$ (if $W \neq \varnothing$ ). This completes the proof of Theorem 3 .

\section{The Structures of $\operatorname{Min} G_{E}(n, 3,4,1)$}

Consider the graphs displayed in Figure 5 below. For $m>4$ we obtain the class $M_{j}^{2 m}(j=1,2, \ldots, 6)$ from the class $M_{j}^{2 m-2}$ by adding an $\left(x_{1}, x_{2}\right)$-path of length 3 . The class $M_{7}^{2 m}$ is obtained from the class $M_{1}^{2 m^{\prime}}, m^{\prime}<m$, by adding $m-m^{\prime}$ triangles with $x_{1}$ being a common vertex in all triangles and the other two being new vertices. It is easily seen that these graphs belong to the class $G_{E}(n, 3,4,1)$ and have $\left[\frac{1}{2}(3 n-4)\right]$ edges. It turns out that these graphs together with the graphs $K_{1}^{2 m+1}$ will exhaust all the extremal graphs in the class $G_{E}(n, 3,4,1)$ except for $n=8,10$ and 12 for which the graphs of Figure 6 also belong to the class $G_{E}(n, 3,4,1)$ and have $\left[\frac{1}{2}(3 n-4)\right]$ edges.

We note that the graphs of $M_{7}^{2 m}$ are the only one of the above graphs having a cut vertex. Suppose that $G$ has a cut vertex, $c$ say, and $G=G_{1} \cup G_{2}$, where
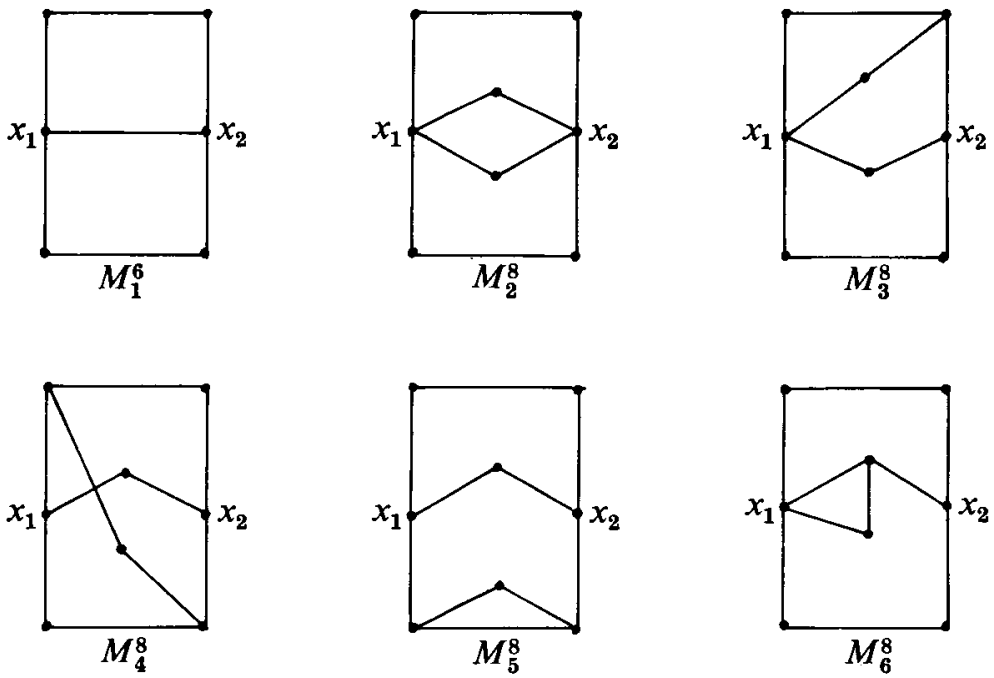

FiguRe 5. 


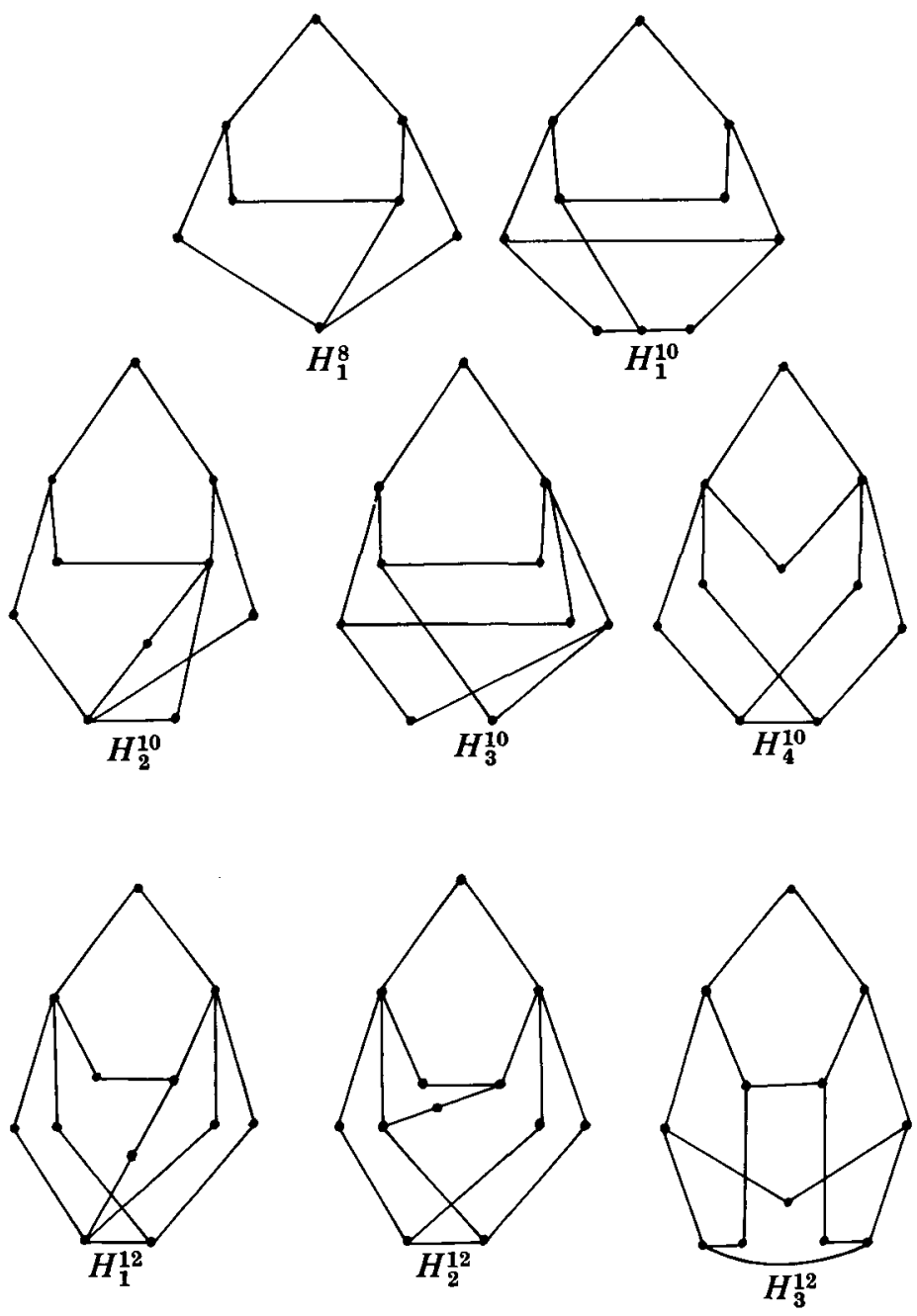

Figure 6.

$V\left(G_{1}\right) \cap V\left(G_{2}\right)=c$. Let $n_{1}=\left|V\left(G_{1}-c\right)\right|$ and $n_{2}=\left|V\left(G_{2}\right)\right|$ so that $n=n_{1}+n_{2}$. Since $D(G) \leqslant 3$, it can be supposed that every vertex of $G_{1}-c$ is adjacent to $c$. Hence the subgraph spanned by $G_{1}$ has $\geqslant \frac{3}{2} n_{1}$ edges. Bollobás (1968b) proved that for $\lambda \geqslant 6, M_{E}(n, 3, \lambda, 1)=\left[\frac{1}{2}(3 n-6)\right]$. This together with observation (1) of Section 2 gives

$$
\left[\frac{1}{2}(3 n-6)\right] \leqslant M_{E}(n, 3,4,1) \leqslant\left[\frac{1}{2}(3 n-4)\right] .
$$

Therefore $M_{K}(n, 3,4,1)=\left[\frac{1}{2}(3 n-k)\right.$, where $k$ is an integer taking on values between 4 and 6 . Now the subgraph obtained by deleting the $n_{1}$ vertices of $G_{1}-c$ belongs 
to the class $G_{E}(n, 3,4,1)$ and has at least

$$
M-\frac{3}{2} n_{1}=\left[\frac{1}{2}(3 n-k)\right]-\frac{3}{2} n_{1}=\left[\frac{1}{2}\left(3 n_{2}-k\right)\right] \text { edges. }
$$

We can therefore restrict our structural study of $\operatorname{Min} G_{E}(n, 3,4,1)$ to the case where $G$ has no cut vertex, since if it had, then we can delete vertices and once we have a basic graph then we can add vertices to it so as to form a cut vertex.

We notice that Lemma 2 with its three corollaries holds for the edge case. Also, equations (4)-(9) (with equality possible in (7)) hold. It seems natural to expect that the proof of Lemma 3 can be used to establish an edge analogue of that lemma. This is in fact the case. However, here the problem becomes much more complex as there are more cases to be considered (for example, we need to consider the case $d_{1}=\frac{1}{2}$ ). Consequently we do not give the details here but simply state the results, for full details we refer to Caccetta (1976b).

LEMma 5. $G$ possesses two adjacent vertices of degree 2 for every $n \geqslant 5$ except possibly $n=8,10$ and 12 . Furthermore, for these values of $n$ the only other possible structures are the graphs $H_{1}^{8}, H_{j}^{10}(j=1,2,3,4)$ and $H_{j}^{12}(j=1,2,3)$ of Figure 6.

With only minor modification the proof of Lemma 1 can be used to prove,

LEMMA 6. If $G$ has no cut vertex then $M_{E}(n, 3,4,1)=\left[\frac{1}{2}(3 n-5)\right]$, and the graphs of $H_{1}^{8}, H_{j}^{10}(j=1,2,3,4), H_{j}^{12}(j=1,2,3), M_{j}^{2 m}(j=1,2, \ldots, 6)$ and $K_{1}^{2 m+1}$ are the elements of $\operatorname{Min} G_{E}(n, 3,4,1)$ for $n=8,10,12,2 m$ and $2 m+1$ respectively.

If $G$ has a cut vertex it must be $x_{1}$ or $x_{4}$ since $D(G) \leqslant 3$. It is easily seen that the basic graph $M_{1}^{6}$ is the only graph which can have a cut vertex. This together with Lemma 6 gives

THEOREM 3. For every $n \geqslant 4, M_{E}(n, 3,4,1)=\left[\frac{1}{2}(3 n-4)\right]$ and the graphs of $H_{1}^{8}, H_{j}^{10}$ $(j=1,2,3,4), H_{j}^{12}(j=1,2,3), M_{j}^{2 m}(j=1,2, \ldots, 7)$ and $M_{1}^{2 m+1}$ are the elements of $\operatorname{Min} G_{E}(n, 3,4,1)$ for $n=8,10,12,2 m$ and $2 m+1$ respectively.

\section{The structures of $\operatorname{Min} G_{E}(n, 3, \lambda, 1), \lambda \geqslant 5$.}

Bollobás (1968b) proved that if $\lambda \geqslant 6$ and $n \geqslant 6$, then $M_{E}(n, 3, \lambda, 1)=\left[\frac{1}{2}(3 n-6)\right]$. In the following we show that the structures of $\operatorname{Min} G_{E}(n, 3, \lambda, 1), \lambda \geqslant 6$, coincide with the structures of $\operatorname{Min} G_{E}\left(n, 3, \lambda^{\prime}, 1\right), \lambda^{\prime} \geqslant 5$.

As in the previous section we can restrict our structural study of extremal graphs to the case when $G$ has no cut vertex. Then obviously the graphs of $L_{j}^{2 m+1}(j=1,2)$ and $L_{j}^{2 m}(j=1,2,3,4)$ defined in Section 4 are the only extremal graphs. From 
these graphs it is clear that if $G$ has a cut vertex then it should be either $x_{1}$ or $x_{4}$. In either case it is easily seen that $D(G)>3$ if $x_{1}$ or $x_{4}$ is a cut vertex. Consequently,

THEOREM 4. If $\lambda \geqslant 6$ and $n \geqslant 6$, then $M_{E}(n, 3, \lambda, 1)=\left[\frac{1}{2}(3 n-6)\right]$, and the elements of $\operatorname{Min} G_{E}(n, 3, \lambda, 1)$ and $\operatorname{Min} G_{V}(n, 3, \lambda, 1)$ coincide.

We now consider the case $\lambda=5$. The following constructions show that $M_{E}(n, 3,5,1) \leqslant\left[\frac{1}{2}(3 n-5)\right]$. Denote by $P_{j}^{2 m}(j=1,2)$ the class obtained from the class $L_{j}^{2 m-1}$ by adding a vertex $x_{2 m}$ and connecting it to $x_{1}$ and one of $x_{2}$ or $x_{3}$ (see Figure 3). The class $K_{4}^{2 m+1}\left(K_{5}^{2 m+1}\right)$ is obtained from the class $P_{1}^{2 m}\left(P_{2}^{2 m}\right)$ by adding a vertex $x_{2 m+1}$, joined to each vertex in the neighbour set of $x_{2 m}$. The class $K_{6}^{2 m+1}$ is obtained from the class $K_{1}^{2 m^{\prime}+1}, m^{\prime}<m$, by adding $m-m^{\prime}$ triangles with $x_{1}$ being a common vertex in all triangles and the other two being new vertices. Put $P_{3}^{2 m}=K^{2 m}$. Clearly the graphs of $P_{j}^{2 m}(j=1,2,3)$ and $K_{j}^{2 m+1}(j=1,2, \ldots, 6)$ belong to the class $G_{E}(n, 3,5,1)$, for $n=2 m$ and $n=2 m+1$, respectively, and they have $\left[\frac{1}{2}(3 n-5)\right]$ edges.

It follows from observation (1) of Section 2 and Theorem 4 above that

$$
\left[\frac{1}{2}(3 n-6)\right] \leqslant M_{E}(n, 3,5,1) \leqslant\left[\frac{1}{2}(3 n-5)\right] .
$$

As in the case $\lambda=4$ we can restrict our structural study of extremal graphs to the case when $G$ has no cut vertex. Therefore $G$ belongs to the class $G_{\nabla}\left(n, 3, \lambda^{\prime}, 1\right)$ for some $\lambda^{\prime}$. If $\lambda^{\prime}=4$ then, by Theorem $1, M \geqslant\left[\frac{1}{2}(3 n-5)\right]$. If $\lambda^{\prime} \geqslant 5$ then, since for $n$ odd the graphs of $\operatorname{Min} G_{V}\left(n, 3, \lambda^{\prime}, 1\right)$ do not belong to the class $G_{E}(n, 3,5,1)$ (see Figure 3$), M \geqslant\left[\frac{1}{2}(3 n-5)\right]$. Hence $M_{E}(n, 3,5,1)=\left[\frac{1}{2}(3 n-5)\right]$.

With only minor modification the proof of Lemma 3 can be used to prove

Lemma 7. Let $G \in \operatorname{Min} G_{E}(n, 3,5,1)$. Then $G$ possesses two adjacent vertices of degree 2 for every $n \geqslant 5$ except possibly $n=9$ for which case the only other possible structure is the graph $H^{9}$ of Figure 2.

We can now easily establish the following theorem.

THEOREM 5. If $n \geqslant 5$, then $M_{E}(n, 3,5,1)=\left[\frac{1}{2}(3 n-5)\right]$, and the graphs of $H^{9}, P_{j}^{2 m}$ $(j=1,2,3)$ and $K_{j}^{2 m+1}(j=1,2, \ldots, 6)$ are the elements of $\operatorname{Min} G_{E}(n, 3,5,1)$ when $n=9, n=2 m$ and $n=2 m+1$, respectively.

\section{References}

B. Bollobás (1968a), 'A problem in the theory of communication networks', Acta Math. Acad. Sci. Hungar. 19, 75-80.

B. Bollobás (1968b), 'Graphs of given diameter', in: Proceedings of Colloquium held at Tihany 1968 (ed. P. Erdos and G. Katona), pp. 29-36. 
B. Bolloods (1976), 'Graphs with given diameter and minimal degree', Ars Combinatoria 2, 3-9.

B. Bollobás and F. Harary (1976), 'Extremal graphs with given diameter and connectivity', Ars Combinatoria 1, 281-296.

J. A. Bondy and U. S. R. Murty (1976), Graph theory with applications (Macmillan, London).

L. Caccetta (1976a), 'A problem in extremal graph theory', Ars Combinatoria 2, 33-56.

L. Caccetta (1976b), 'Characterization of extremal graphs with given diameter and connectivity -part I' (University of Waterloo Research Report CORR 76-42).

L. Caccetta (1977), 'On extremal graphs', in: Proceedings of Eighth South-eastern Conference on Combinatorics, Graph Theory and Computing (Congressus Numerantium XIX) (ed. F. Hoffman et al.) (Utilitas Mathematica, Winnipeg), pp. 125-137.

L. Caccetta (1978), Graph theory and graphical enumeration with applications to the physical sciences and social sciences (ed. F. Harary) (New York Academy of Sciences), pp. 76-94.

\author{
Department of Mathematics \\ University of Waterloo \\ Ontario, N2L 3G1 \\ Canada
}

